\title{
Comportamento Locomotor de Crianças e Adultos Jovens em Ambiente Doméstico Simulado ${ }^{1}$
}

\author{
Lilian Teresa Bucken Gobbi² \\ Jean José Silva \\ Ana Clara Souza Paiva \\ Priscila Elaine Scabello \\ Universidade Estadual Paulista - Campus Rio Claro
}

\begin{abstract}
RESUMO - Observamos o comportamento locomotor de 40 crianças (de 5 a 8 anos) e de 40 adultos jovens (de 19 a 28 anos) frente a restrições ambientais, que percorreram dois circuitos em condições de luminosidade total e reduzida e transporte de carga (sem carga, $3 \%$ e 7\% da massa corporal), andando o mais rápido possível e evitando contato com objetos. O tempo gasto na tarefa foi obtido por meio de um cronômetro digital, em três tentativas por condição. ANOVA para três fatores (circuito $\mathrm{x}$ iluminação x carga) revelou que: as crianças demoraram mais tempo para realizar a tarefa quando carga foi adicionada; crianças mais jovens percorreram o circuito em tempo maior que crianças mais velhas; as condições não alteraram a locomoção dos adultos. Concluímos que crianças necessitam integrar as informações sensoriais e acoplá-las ao sistema efetor. Programas de intervenção são necessários para facilitar essa integração sem restrição da mobilidade das crianças.
\end{abstract}

Palavras-chave: locomoção; restrições do ambiente; desenvolvimento motor.

\section{Locomotor Behavior of Children and Young Adults in Simulated Domestic Environment}

\begin{abstract}
In order to observe the locomotors behavior of children and young adults facing environmental constraints, 40 children (from 5 to 8 years old) and 40 young adults (from 19 to 28 years old) went through two circuits under full and reduced luminosity and cargo transportation (no cargo, $3 \%$ and $7 \%$ of body mass) conditions, walking as fast as possible and avoiding contact with objects. The time spent to complete the task was obtained by means of a digital stopwatch, in three trials per condition. Three-way ANOVA factors (circuit $\mathrm{x}$ illumination $\mathrm{x}$ cargo) revealed that: children spent more time to complete the task when cargo was added; younger children were slower than older children; adults' locomotion were not affected by the conditions. It can be concluded that children need to integrate the sensory information and couple them with the effectors system. Intervention programs are necessary to facilitate this integration without restricting children's mobility.
\end{abstract}

Key words: locomotion; environment constraints; motor development.

A criança vive em ambiente complexo, com objetos e mobiliários domésticos que são proporcionais à escala corporal dos adultos, e não está com seu sistema perceptivo e motor completamente maduro e integrado. Paralelamente, a criança apresenta equilíbrio e postura em desenvolvimento e, com isso, demonstra dificuldade de mobilidade em terrenos irregulares (Gobbi, 1997; Gobbi \& Patla, 1997). Maturação e experiências no ambiente são os componentes responsáveis para que o desenvolvimento da mobilidade ocorra (Adolph, Eppler, Marin, Weise \& Clearfield, 2000). Adultos jovens, por outro lado, apresentam seus sistemas perceptivo e motor integralmente desenvolvidos e interagem em ambientes construídos de acordo com sua escala corporal. Portanto, adultos jovens não devem apresentar dificuldades locomotoras em ambientes domésticos.

1 Os autores agradecem a Cândida Taís Gonçalves e Veronica Miyasikeda Silva pela colaboração na coleta dos dados e ao CNPq pela bolsa de Iniciação Científica (CNPq - 520287/98-3) ao segundo autor.

2 Endereço: Avenida 24A, $\mathrm{n}^{\circ}$ 1515, Bela Vista, Rio Claro, SP, Brasil 13506-900.E-mail: ltbgobbi@rc.unesp.br
Quando a criança está aprendendo a andar, pais retiram objetos do ambiente que possam causar alguma colisão, mas após esse período a criança deve interagir em um ambiente complexo, com obstáculos como brinquedos pelo chão. Os sistemas sensoriais isoladamente não estão maduros nem seletivos, ou seja, a criança não consegue captar apenas as informações relevantes. Similarmente, o sistema perceptivo não consegue integrar as informações provenientes dos diferentes sistemas sensoriais. Além disso, o sistema efetor, nesse período, sofre intensas mudanças antropométricas. Essas características formam o padrão locomotor infantil e dificultam a análise isolada de cada componente (Joh \& Adolph, 2006).

A locomoção sobre obstáculos requer a utilização de informação sensorial combinada para modular o sistema efetor na ultrapassagem. Na vida diária observa-se que as pessoas ajustam seus passos e focalizam a atenção quando é necessário ultrapassar o obstáculo. Em contraste, o andar normal em um meio ambiente regular requer menos atenção dirigida às informações sensoriais e as pessoas, então, são aptas a combinar outras tarefas durante o andar (Gobbi \& Patla, 1997). Assim, o andar é um comportamento complexo, 
que envolve todo o corpo, requer a coordenação de muitos músculos e articulações, além do sistema sensorial, principalmente quando o ambiente oferece restrições.

O sistema visual extrai basicamente dois tipos de informações necessárias para promover uma locomoção segura sobre obstáculos e com custo energético baixo: informação exteroceptiva e informação exproprioceptiva (Gibson, 1979, 1982). Informação exteroceptiva refere-se à identificação da localização e formas dos objetos e superfície no ambiente. Durante a locomoção em terrenos irregulares, os sinais exteroceptivos são necessários para planejar com antecedência as mudanças adaptativas a serem executadas (Moraes, Lewis \& Patla, 2004; Moraes \& Patla, 2006; Pryde, Roy \& Patla, 1997). "Crianças pequenas podem detectar as propriedades dos objetos e eventos através da exploração e assim aprender como sintonizar suas ações apropriadamente" (Adolph, Eppler \& Gibson, 1993, p. 73). Informação exproprioceptiva refere-se à identificação das partes do corpo relativas umas às outras e relativas aos objetos e eventos no ambiente (Gobbi, Menuchi, Uehara \& Silva, 2003; Patla, Prentice \& Gobbi, 1996). Os sinais exproprioceptivos, que fornecem informação particularmente sobre a velocidade e orientação do corpo e de seus segmentos, são extraídos pela visão (Lee \& Young, 1986).

Para uma correta identificação dos objetos e eventos no meio ambiente, as estruturas de luz refletidas pelos objetos e superfícies devem ser captadas e, então, percebidas. Durante os primeiros cinco anos de vida, a sensibilidade ao contraste entre essas estruturas de luz melhora acentuadamente e atinge os níveis adultos (Movshon \& Kiorpes, 1988). Se as funções visuais não estão maduras na idade pré-escolar, a extração das informações exteroceptivas e exproprioceptivas pode estar comprometida, evidenciando que os erros de julgamento ocorridos possam ser atribuídos ao processo de maturação. Estudos mostram que há diferença entre as idades quanto à locomoção e ultrapassagem de obstáculos, pois os mais velhos utilizam os sinais exteroceptivos e exproprioceptivos de melhor maneira para manter o equilíbrio (Gobbi, 1997). Essas mudanças ocorrem ao longo dos anos mostrando que, com o aumento da idade, a criança pode tornar-se mais experiente e mais preparada para interagir com o ambiente e, portanto, melhorar sua mobilidade.

A criança entre 5 e 8 anos de idade, faixa etária abrangida neste estudo, encontra-se em uma fase de transição no que se refere às características locomotoras. Por volta dos 5 e 6 anos, a criança deve apresentar características maduras nos componentes do andar (Gallahue \& Ozmun, 1998), normalmente observados em pisos regulares e desobstruídos. A partir dessa idade, e quando expostos a ambientes complexos, o padrão locomotor das crianças altera-se basicamente em dois sentidos: a) ocorre um retrocesso no padrão de andar, no qual observam-se características imaturas (Ferreira, Marins \& Gobbi, 1999; Lima, Secco, Miyasike \& Gobbi, 2001) e b) surgem tentativas de aprimoramento, de busca de soluções novas para o desafio imposto pelo ambiente, em que a criança tenta descobrir e empregar diferentes estratégias locomotoras adaptativas (Gobbi, 1997).

Pryde e cols. (1997) investigaram as mudanças com relação à idade (entre 5 e 16 anos) que ocorrem na habilidade para locomover e manobrar em um ambiente complexo com controle e precisão em três tarefas locomotoras: 1) pista reta; 2) circuito em forma de $\mathrm{U}$ sem objetos e 3) circuito em forma de U com 33 obstáculos. Os obstáculos incluídos na terceira tarefa eram de espuma, variando em tamanho, forma (sete retângulos achatados, cinco retângulos, 10 cilindros, nove tubos e dois blocos) e cor. Os obstáculos foram dispostos em posições horizontais, verticais e diagonais, de forma que as crianças deveriam desviar de alguns deles e passar por cima de outros. O número de erros (pisar fora das linhas ou tocar em qualquer obstáculo), de supercompensações (elevação demasiada da perna para passar sobre um obstáculo ou exagerada flexão do tronco para passar sob um obstáculo) e o tempo total para percorrer os circuitos foram calculados para cada tentativa. A análise de tempo de movimento revelou: a) as crianças mais jovens, independentemente da tarefa, demoraram mais tempo para percorrer os circuitos do que as mais velhas e um b) aumento de tempo de movimento quando aumentou a complexidade da tarefa. Um efeito de interação entre grupo e condição foi também revelado. Uma análise apenas nos efeitos principais de grupo indicou efeito da idade somente no circuito com obstáculos, ou seja, as crianças mais jovens percorreram o circuito de obstáculos em tempo maior do que as crianças mais velhas. A quantidade de erros cometidos não foi suficiente para comparar os circuitos reto e curvilíneo. A análise dos erros no circuito de obstáculo revelou que as crianças mais jovens cometeram um número significativamente maior de erros e demonstraram mais supercompensações do que as crianças mais velhas.

Esses resultados permitiram aos autores concluir que existem mudanças relacionadas à idade na habilidade locomotora, dependendo da natureza da tarefa. A idade teve seu maior efeito no circuito mais complexo, o que mostra que o sistema sensório-motor das crianças mais novas ainda não está integrado. Os elementos dos sistemas necessários para desviar obstáculos, que são o visual, cinestésico e efetor, estão em processo de refinamento e mudanças. Obviamente, os requerimentos para o perfeito controle do equilíbrio e da postura ainda não foram atingidos.

Os resultados do estudo de Pryde e cols. (1997) desencadearam questões que foram endereçadas no presente estudo. Os primeiros questionamentos referem-se à idade, ou seja, como crianças um pouco mais jovens (entre 5 e 6 anos) responderiam à complexidade do ambiente? Se as crianças fossem distribuídas em faixas etárias menos abrangentes poder-se-ia observar mudanças no comportamento locomotor? Um segundo grupo de questionamentos refere-se às características do circuito, ou seja, objetos que potencialmente podem causar danos, mas que são encontrados no ambiente doméstico e, portanto, apresentam significado para a criança, seriam capazes de desencadear mudanças no comportamento locomotor? Um terceiro grupo de questões refere-se à contribuição dos sistemas sensoriais na modulação do comportamento locomotor, ou seja, poderia a variação na luminosidade do ambiente provocar mudanças locomotoras? Como o transporte de carga poderia influenciar no comportamento locomotor? Espera-se que adultos jovens não sejam influenciados pela iluminação e pelo transporte de carga. 
Nesse contexto, o objetivo deste estudo foi observar o comportamento locomotor de crianças e adultos jovens frente a restrições ambientais (iluminação e transporte de carga) em dois circuitos. Na construção dos circuitos, objetos cotidianos foram utilizados para tornar o ambiente experimental o mais próximo possível do ambiente doméstico.

\section{Método}

\section{Participantes}

Participaram do estudo 40 crianças, com idade entre 5 a 8 anos, de ambos os sexos; e 40 universitários com idade entre 19 a 28 anos, de ambos os sexos.

As crianças foram distribuídas em quatro grupos por idade: Grupo1 - 5 anos: sete crianças (quatro do sexo masculino e três do sexo feminino); Grupo $2-6$ anos: 13 crianças (seis do sexo masculino e sete do sexo feminino); Grupo 3 - 7 anos: 10 crianças (oito do sexo masculino e dois do sexo feminino); Grupo 4 - 8 anos: 10 crianças (sete do sexo masculino e três do sexo feminino). Os adultos jovens não foram distribuídos em grupos.

\section{Procedimentos}

Após obtenção do termo de consentimento assinado pelos pais, as crianças foram convidadas a comparecer nas dependências do laboratório. Adultos jovens também consentiram na participação no estudo.

A tarefa realizada consistia em andar o mais rápido possível por um circuito em forma de "U", de aproximadamente 25 metros de comprimento, numa superfície com carpete e com objetos domésticos, como: vassoura, balde, cadeira, cesto de lixo, brinquedos, etc. Um total de 33 objetos foi disposto no circuito. O mesmo circuito foi utilizado por todos os participantes. A Figura 1 apresenta uma representação gráfica do circuito e dos objetos dispostos; os objetos assinalados com setas representam objetos suspensos.

Considerando-se a disposição dos objetos no circuito, foi estabelecido que o circuito 1 indicava início pela direita e término pela esquerda. O circuito 2 iniciava pela esquerda e terminava na direita. O circuito 1 apresentava, no início, objetos que alteram a superfície de apoio, como: degraus, rampa descendente e colchões alterando a densidade do piso. O circuito 2 apresentava, no início, apenas objetos colocados sobre a superfície e que requeriam comportamentos de desvio.

Para poder verificar as influências da percepção visual no comportamento locomotor, foram estabelecidas duas condições de luminosidade: total (aproximadamente 100 a 300 lux) e reduzida (3 a 10 lux). A intensidade da luz foi medida no início da coleta de cada participante por meio de um Luxímetro digital (Minipa/MLM - 1332). As mesmas condições de iluminação foram apresentadas para as crianças e para os adultos jovens.

Para verificar a influência do transporte de carga, três condições com carga foram estabelecidas, utilizando uma mochila para as crianças e sacolas de compras para os adul-

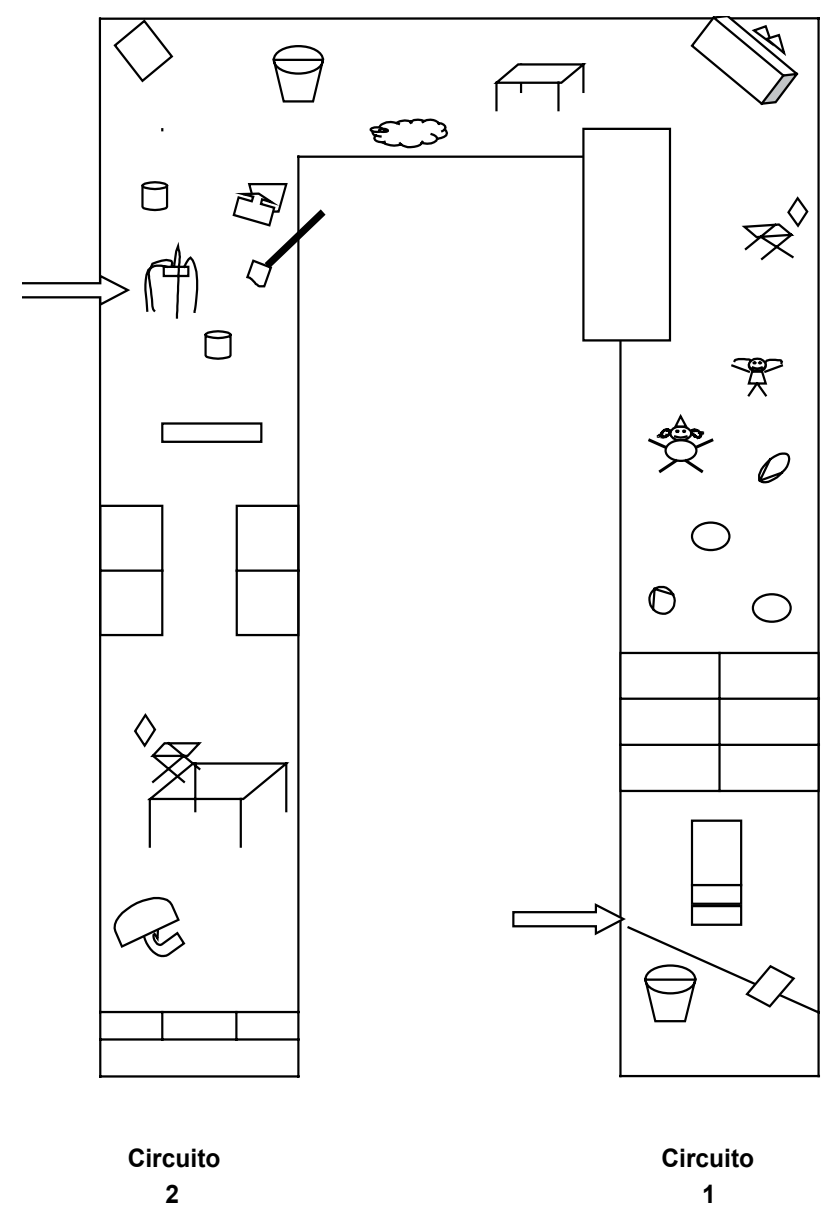

Figura 1. Representação gráfica do circuito de objetos domésticos (veja o texto para maiores detalhes).

tos jovens: com 3\% da massa corporal, com $7 \%$ da massa corporal e sem carga. Dessa forma, a carga foi personalizada para cada participante.

A combinação de três tentativas para cada condição experimental resultou em 36 tentativas randomizadas por participante. Intervalos de descanso foram concedidos sempre que solicitados pelo participante ou sempre que o experimentador observou serem necessários. Cada participante foi instruído a percorrer o circuito andando, o mais rápido possível, e evitando ter contato com os objetos ou sair da área que limitava o circuito. Em cada tentativa foram coletados: o tempo gasto para percorrer o circuito e o número de erros.

\section{Análise de dados}

O tempo gasto para percorrer o circuito foi obtido para cada tentativa por meio de cronômetro digital de precisão de centésimos de segundos, sendo registrado na ficha de coleta de dados. Para o número de erros (contatos com os objetos e/ou pisar fora do circuito), foi observado o objeto específico e registrado na ficha de coleta de dados. Os dados foram analisados por meio de Análise de Variância Multifatorial, com um nível de significância pré-estabelecido de $p<0,05$. 


\section{Resultados}

Em relação às crianças, a análise de variância revelou efeito principal de carga para a média do tempo gasto para percorrer o circuito $\left(F_{2,480}=13,805 ; p \leq 0,002\right)$. O teste post-hoc de Tukey evidenciou que o acréscimo de carga aumenta o tempo para percorrer o circuito, sendo que não houve diferença entre as cargas de $3 \%$ e $7 \%$. As crianças percorreram o circuito mais rápido quando não estavam transportando peso adicional (Figura 2).

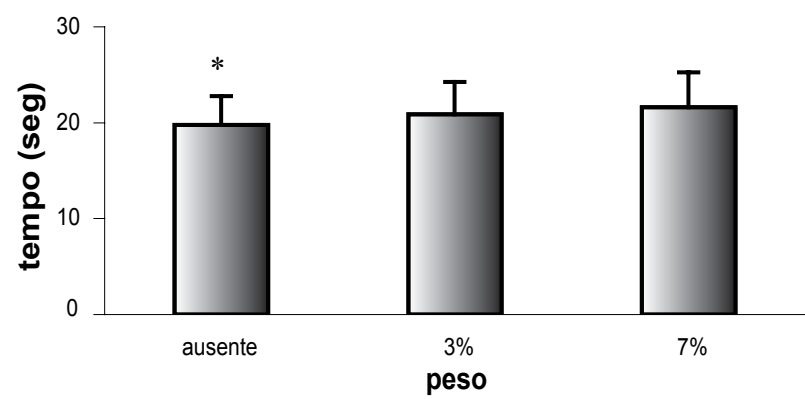

Figura 2. Representação gráfica das médias e desvios-padrão do tempo por carga $(*=$ diferença significativa).

Para média de tempo das crianças, a análise de variância também evidenciou efeito principal de idade $\left(F_{3,480}=43,43\right.$; $p \leq 0,001)$. O teste post-hoc de Tukey demonstrou que as crianças de 5 e 6 anos são iguais entre elas, mas diferentes das demais idades, ou seja, exceto entre 5 e 6 anos, o aumento da idade diminui o tempo de percurso (Figura 3 ). Nenhuma das interações entre idade, luminosidade, transporte de carga e circuito mostrou-se significativa.

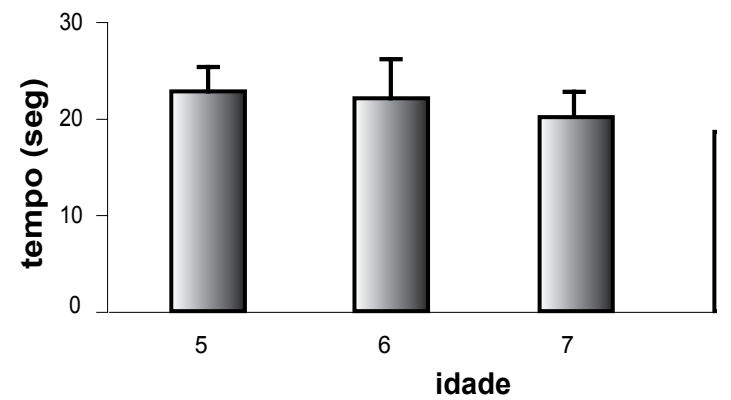

Figura 3. Representação gráfica das médias e desvios-padrão do tempo por idade.

Em relação aos adultos, quanto ao tempo gasto para percorrer o circuito, a ANOVA não detectou efeitos principais de circuito $\left(F_{1.480}=1,015 ; p=0,314\right)$, iluminação $\left(F_{1,480}=1,012 ; p=0,315\right)$, $\operatorname{carga}\left(F_{2,480}=1,002 ; p=0,368\right) \mathrm{e}$ interações entre os fatores.

Para média de contatos das crianças nos objetos, houve efeito principal de idade $\left(F_{3,480}=23,056 ; p<0,001\right)$. O teste post-hoc de Tukey evidenciou que as crianças de 5 e 6 anos tiveram mais contato com os objetos do circuito e que apenas as crianças mais jovens são estatisticamente iguais entre si e diferentes das crianças mais velhas. Quanto mais velha a criança, menos erros ela comete ao percorrer o

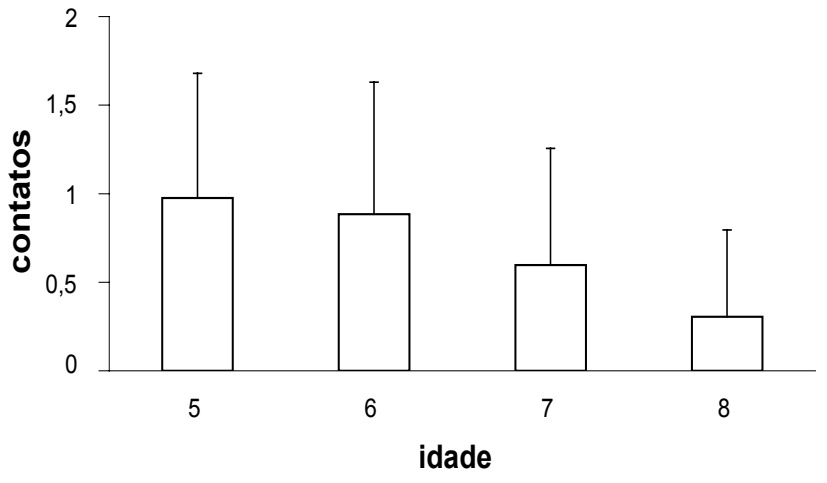

Figura 4. Representação gráfica das médias e desvios-padrão para o número de contatos por grupo de idade.

circuito (Figura 4). Nenhuma interação entre idade, luminosidade, transporte de carga e circuito atingiu o nível de significância crítico.

Em relação aos adultos, quanto aos contatos com os objetos do circuito, a ANOVA não detectou efeitos principais de circuito $\left(F_{1,480}=0,229 ; p=0,633\right)$, iluminação $\left(F_{1,480}=1,685 ; p=0,195\right)$, carga $\left(F_{2,480}=0,621 ; p=0,538\right) \mathrm{e}$ interações entre os fatores.

\section{Discussão}

A discussão dos resultados deste estudo está organizada de forma a responder aos questionamentos propostos. A execução da tarefa requereu dos participantes a percepção dos objetos no circuito, a elaboração da melhor rota e a modulação do sistema efetor.

$\mathrm{O}$ primeiro conjunto de questionamentos refere-se à idade, ou seja, como crianças um pouco mais jovens (entre 5 e 6 anos) responderiam à complexidade do ambiente e se as crianças fossem divididas em faixas etárias menos abrangentes, poder-se-ia observar mudanças no comportamento locomotor? Os resultados demonstraram que, tanto em relação ao tempo gasto para percorrer o circuito como em relação ao número de erros, as crianças de 5 e 6 anos foram semelhantes entre si mas diferentes dos participantes das demais faixas etárias. Em relação à divisão dos participantes em faixas etárias menores, os resultados também evidenciaram efeitos da idade. A idade também esteve presente na combinação das variáveis explicando a variabilidade do comportamento locomotor, predizendo o tempo para percorrer o circuito e o número de erros.

Esses resultados corroboram com as descobertas de Gobbi (1997), Ferreira e cols. (1999) e Lima e cols. (2001). A idade cronológica, ainda que sendo considerada como um indicador pouco eficiente para revelar mudanças comportamentais, evidenciou, no presente estudo, que quando variáveis de desempenho como tempo e erro são consideradas, mudanças podem ser observadas. Embutidos na idade cronológica estão os efeitos da maturação e da experiência. Crianças mais jovens não poderiam revelar o mesmo comportamento de crianças mais velhas em função da maturação de cada sistema (perceptivo e motor), da integração desses sistemas e do próprio desenvolvimento cognitivo, especialmente na elaboração da rota para percorrer o circuito. 
Em relação às características do circuito, ou seja, objetos que potencialmente podem causar danos mas que são encontrados no ambiente doméstico e, portanto, apresentam significado para a criança, seriam capazes de desencadear mudanças no comportamento locomotor? As diferenças esperadas entre os circuitos, especialmente pelas diferenças na disposição dos objetos, não foram observadas. As crianças foram capazes de realizar a tarefa independentemente da disposição dos objetos, evidenciando que o hábito de conviver com mobiliário não proporcional às suas características antropométricas pode ter favorecido o desempenho locomotor.

Em relação ao terceiro grupo de questões referente à contribuição dos sistemas sensoriais na modulação do comportamento locomotor, ou seja, poderia a variação na luminosidade do ambiente provocar mudanças locomotoras e como o transporte de carga poderia influenciar no comportamento locomotor, os resultados evidenciaram apenas efeito do transporte de carga no tempo para percorrer o circuito. Quanto à luminosidade, a quantidade de luz disponível na condição com iluminação restrita permitia a visualização do ambiente. Paralelamente, a adaptação ao claro e ao escuro depende da quantidade de rodopsina disponível (Guyton, 1988; Schmidt, 1980), sendo que crianças, na faixa etária deste estudo, possuem quantidades de rodopsina similares ao adulto.

O transporte de carga contribuiu para perturbar o controle do equilíbrio e da postura. Durante o andar, é necessária a interação entre vários sistemas: nervoso, muscular, esquelético, articular, além de muitos outros componentes. Existe uma troca de energia a cada passada entre o executante e o ambiente em que a ação está ocorrendo. Mais especificamente, quando o pé está em contato com o solo, essa troca de energia precisa ser inserida no sistema. O executante precisa aplicar a força para sustentar seu corpo ereto e também para propulsioná-lo à frente. Em superfícies diferentes, quantidades diferentes de energia devem ser utilizadas e transferidas ao ambiente acomodando as peculiaridades daquela específica superfície (Barela, 1997).

Para uma locomoção bem sucedida, são indispensáveis um grau suficiente de força, o desenvolvimento dos mecanismos de equilíbrio sensório-motor e a aquisição de novas coordenações neuromotoras. A exploração de novos modos de locomoção é muito grande durante a primeira infância (Adolph, 1995; Eckert, 1993). O equilíbrio, base de toda a coordenação dinâmica global, é a noção de distribuição do peso em relação a um espaço e um tempo e em relação ao eixo de gravidade. O equilíbrio depende essencialmente do sistema labiríntico e do sistema plantar, podendo ser estático ou dinâmico. O controle do equilíbrio implica o controle da postura. O equilíbrio dinâmico está em estreita relação com a constituição estato-ponderal, com as funções tônico-motoras, com os membros e os órgãos, tanto os sensoriais como os motores. O equilíbrio está em estreita ligação com a área temporal e estímulos vestibulares são extremamente necessários à manutenção do equilíbrio. O aparelho vestibular é o órgão sensorial que detecta as sensações relacionadas com o equilíbrio. Uma de suas principais funções relaciona-se à manutenção do equilíbrio vertical, necessário para a manutenção da posição ereta. $\mathrm{Na}$ evolução psicomotora da criança é necessário que ela tome consciência do seu contato com o solo e com a mobilidade da articulação do tornozelo para uma boa progressão do equilíbrio (Bueno, 1998).

A aplicação de uma carga de peso extracorporal altera tanto a quantidade de força a ser aplicada como o controle do equilíbrio, visto que o transporte de carga, com mochila, move o centro de gravidade do corpo para trás. O centro de gravidade corporal está relacionado com a constituição física de cada indivíduo, e a sua posição varia a cada momento em que o indivíduo realiza um movimento. Crianças possuem um centro de gravidade elevado devido ao tamanho desproporcional entre membros inferiores, tronco e cabeça. Quanto mais jovem é a criança, mais alto estará localizado seu centro de gravidade. Para crianças que estão iniciando a marcha, a cabeça é um grande empecilho no que se refere à manutenção do equilíbrio dinâmico. Essa manutenção resulta da interação entre estruturas especializadas na identificação e processamento de informações exteroceptivas, exproprioceptivas e cinestésicas (Gobbi, 1997; Gobbi \& Patla, 1997).

O processamento das informações tanto visuais como proprioceptivas referentes à superfície de apoio e aos objetos dispostos em um ambiente complexo e a modulação apropriada do sistema efetor demandam um tempo maior nessas condições. Dessa forma, não apenas a maturação neurofisiológica e as experiências motoras devem ser consideradas na análise do equilíbrio, mas também as restrições das tarefas que desencadeiam mudanças locomotoras (Barela, 1997; Gobbi \& Patla, 1997; Newell, 1986; Newell \& Mcdonald, 1992).

Os resultados do presente estudo, quando comparados com os resultados Pryde e cols. (1997), mostram que com o aumento da idade a performance melhora. Isso ocorre devido ao fato de as estruturas importantes na realização da tarefa locomotora estarem mais desenvolvidas. Considerando-se as diferenças quanto às tarefas e às faixas etárias envolvidas nos dois estudos, as conclusões são similares.

Em relação aos adultos jovens, os resultados deste estudo mostraram que a mobilidade em ambiente doméstico desses indivíduos não é influenciada pela iluminação e pelo transporte de carga, confirmando a hipótese levantada. Adultos jovens possuem os sistemas perceptivo e motor completamente desenvolvidos e integrados, de forma que a locomoção em ambiente complexo apresenta-se segura e eficiente.

\section{Conclusão}

Os resultados obtidos neste estudo permitem concluir que:

a) a idade cronológica influencia no desempenho locomotor de crianças entre 5 e 8 anos de idade;

b) a luminosidade do ambiente, nos limites empregados neste estudo, não interfere na realização de tarefa locomotora;

c) o transporte de carga perturba o controle da postura e do equilíbrio e, portanto, interfere no desempenho locomotor de crianças;

d) a luminosidade do ambiente e o transporte de carga não afetam a mobilidade de adultos jovens. 


\section{Referências}

Adolph, K. E. (1995). Psychophysical Assessment of Toddlers' Ability to Cope With Slopes. Journal of Experimental Psychology: Human Perception and Performance, 21(4), 734-750.

Adolph, K. E., Eppler, M. A. \& Gibson, E. J. (1993). Development of perception of affordances. Advances in Infancy Research, 8, 51-98.

Adolph, K. E., Eppler, M. A., Marin, L., Weise, I. B. \& Clearfield, M. W. (2000). Exploration in the service of prospective control. Infant Behavior \& Development, 23, 441-460.

Barela, J. A. (1997). Perspectiva dos Sistemas Dinâmicos: teoria e aplicação no estudo de desenvolvimento motor. Em A. M. Pellegrini (Org.), Coletânea de Estudos: Comportamento Motor 1 (pp. 11-28). São Paulo: Movimento.

Bueno, J. M. (1998). Psicomotricidade Teoria \& Prática: estimulação, educação e reeducação psicomotora com atividades aquáticas. São Paulo: Lovise.

Eckert, H. (1993). Desenvolvimento Motor. São Paulo: Manole.

Ferreira, L. F. S., Marins, F. H. P. \& Gobbi, L. T. B. (1999). Adaptações locomotoras: efeitos antropométricos e da superfície de apoio [Resumo]. Em UFSC e UDESC (Org.), $7^{\circ}$ Congresso de Educação Física e Ciências do Esporte dos Países de Língua Portuguesa, Anais (p. 327). Florianópolis: UFSC, UDESC.

Gallahue, D. L. \& Ozmun, J. C. (1998). Understanding Motor Development: infants, children, adolescents, adults. Boston: WCB McGraw-Hill.

Gibson, J. J. (1979). The ecological approach to visual perception. Boston: Houghton Mifflin.

Gibson, J. J. (1982). Notes on Affordances. Em E. S. Reed \& R. Jones (Orgs.), Reasons for realism (pp. 401-418). Hillsdale: Erlbaum.

Gobbi, L. T. B. (1997). Developmental trends in skilled locomotor behavior over uneven terrain. Tese de Doutorado, University of Waterloo, Waterloo.

Gobbi, L. T. B., Menuchi, M. R. T. P., Uehara, E. T. \& Silva, J. J. (2003). Influência da informação exproprioceptiva na tarefa locomotora com alta demanda de equilíbrio em crianças. Revista Brasileira de Ciência e Movimento, 11, 79-86.

Gobbi, L. T. B. \& Patla, A. E. (1997). Desenvolvimento da locomoção em terrenos irregulares: proposta de um modelo teórico. Em A. M. Pellegrini (Org.), Coletânea de Estudos: Comportamento Motor 1 (pp. 29-44). São Paulo: Movimento.

Guyton, A. C. (1988). Fisiologia Humana. Rio de Janeiro: Guanabara.

Joh, A. S. \& Adolph, K. E. (2006). Learning from falling. Child Development, 77, 89-102.
Lee, D. N. \& Young, D. S. (1986). Gearing action to the environment. Experimental Brain Research, 15, 217-230.

Lima, C. B., Secco, C. R., Miyasike, V. S. \& Gobbi, L. T. B. (2001). Equilíbrio dinâmico: influência das restrições ambientais. Revista Brasileira de Cineantropometria de Desempenho Humano, 3(1), 83-94.

Moraes, R., Lewis, M. A. \& Patla, A. E. (2004). Strategies and determinants for selection of alternate foot placement during human locomotion: influence of spatial and temporal constraints. Experimental Brain Research, 159, 1-13.

Moraes, R. \& Patla, A. E. (2006). Determinants guiding alternate foot placement selection and the behavioral responses are similar when avoiding a real or a virtual obstacle. Experimental Brain Research, 171, 497-510.

Movshon, J. A. \& Kiorpes, L. (1988). Analysis of the development of spatial contrast sensitivity in monkey and human infants. Journal of the Optical Society of America A, 5(12), 21662172.

Newell, K. M. (1986). Constraints on the Development of Coordination. Em M. G. Wade \& H. T. A. Whiting (Orgs.), Motor Development in Children: Aspects of Coordination and Control (pp. 341-360). Netherlands: Martinus Nijhoff Publishers.

Newell, K. M. \& Mcdonald, P. V. (1992). Searching for solutions to the coordination function: learning as exploratory behavior. Em G. E. Stelmach (Org.), Tutorials in Motor Behavior II (pp. 517-532). Amsterdam: Elsevier.

Patla, A. E., Prentice, S. D. \& Gobbi, L. T. B. (1996). Visual control of obstacle avoidance during locomotion: strategies in young children, young and older adults. Em A.-M. Ferrandez \& N. Teasdale (Orgs.), Changes in Sensori-motor Behavior in Aging (pp. 257-277). Amsterdam: Elsevier.

Pryde, K. M., Roy, E. A. \& Patla, A. E. (1997). Age-relate trends in locomotor ability and obstacle avoidance. Human Movement Science, 16, 507-516.

Schmidt, R. F. (1980). Fisiologia Sensorial. São Paulo: EPU, Springer, Edusp.

Recebido em 21.12.2006

Primeira decisão editorial em 03.05.2006

Versão final em 14.08.2006

Aceito em 22.02.2007 